\title{
LED-generated multifocal ERG On- and Off-responses in complete congenital stationary night blindness - a case report
}

\author{
Dorothee Leifert, Margarita G. Todorova, Chrisitan Prünte \& Anja M. Palmowski-Wolfe \\ Department of Ophthamlology, University Hospital Basel, Mittlere Strasse 91, CH-4056, Basel, Switzerland
}

Accepted 7 July 2005

Key words: LED, multifocal ERG, night blindness, on- and off-response

\begin{abstract}
We report on the application of a light emitting diode (LED) screen to elicit multifocal ERG on- and offresponses in a patient presenting with the complete type of congenital stationary night blindness (cCSNB): A 63-years old woman was diagnosed with cCSNB by means of standard ERG procedures and dark adaptometry. To confirm this diagnosis and to investigate topographical differences of on- and off-responses a multifocal approach employing long-duration stimuli was added. Results of mfERG-testing were averaged in three groups (a central area of $7.5^{\circ}$, a ring area of $7.5-21.9^{\circ}$ and a peripheral ring of $21.9-31.1^{\circ}$ ). When compared to normal controls $(n=4)$ on-responses (P1-amplitudes) were severely reduced symmetrically at all eccentricities, while off-responses showed no reduction resulting in an increased off/on-ratio. Furthermore on-latencies of P1 were delayed symmetrically at all eccentricities, whereas off-latencies were normal. To our knowledge this is the first report of multifocal on- and off-responses in a CSNB-patient. Stimulus-generation with a LED-screen provides the advantage of a stable luminance during the long-duration on-phase.
\end{abstract}

\section{Introduction}

Congenital stationary night blindness has been classified by Miyake et al. [1] according to the results of Ganzfeld-ERG testing. Subtypes of this non-progressive disorder presenting with a so-called "negative-shaped" ERG (SchubertBornschein type) after maximal mixed rod and cone stimulus presentation were separated from "non-negative phenotypes", the so-called Riggs type. This type includes mainly members of two large families (in France the Nougaret-family [2] and in Denmark the Rambusch-family) and is of autosomal-dominant inheritance [3]. The Schubert-Bornschein type has been further subdivided into two forms: A so-called complete

Presented in part at the $102^{\text {nd }}$ meeting of the German Academy of Ophthalmology, Berlin (Germany), 22-26 September 2004. form with no rod response under scotopic conditions and under dark adaptometry but with only a slightly reduced cone response (i.e. slightly reduced amplitudes under photopic conditions, but substantially increased implicit times). In contrast, the so-called incomplete form has residual rod function but a more severe decrease of cone function. Since the negative wave form is the result of a more or less normal photoreceptor response combined with a diminished to absent bipolar response the defect has been subscribed to proximal retinal layers.

Several mutations have been identified for the different phenotypes described above: A mutation at the Xp11.4 locus (X-chromosomal-recessive inheritance) coding for nyctalopin, a protein assumed to play a role during the development of on-synapses, has been assigned to the complete Schubert-Bornschein type (also known as cCSNB 
or CSNB type 1) [4, 5]. Another point mutation (Xp11.2 locus, CACNA1F, X-chromosomalrecessive inheritance) affecting the calcium channel of the inner photoreceptor segment was found to result in the incomplete Schubert-Bornschein type (also known as iCSNB or CSNB type 2) by inhibition of the signal transduction to bipolar cells [6]. For the autosomal recessive forms of these two subtypes no mutations have been identified so far. Dominant CSNB-forms have been

Table 1. Ganzfeld-ERG testing (according to ISCEV standards) of the CSNB patient (left-hand side) exhibits a negative-shaped ERG for the mixed maximal rod and cone response, an absent rod response, as well as a slightly reduced amplitude and increased implicit times under photopic conditions (single photopic flash, 30-Hz-flicker) if compared to a representative control (right-hand side)

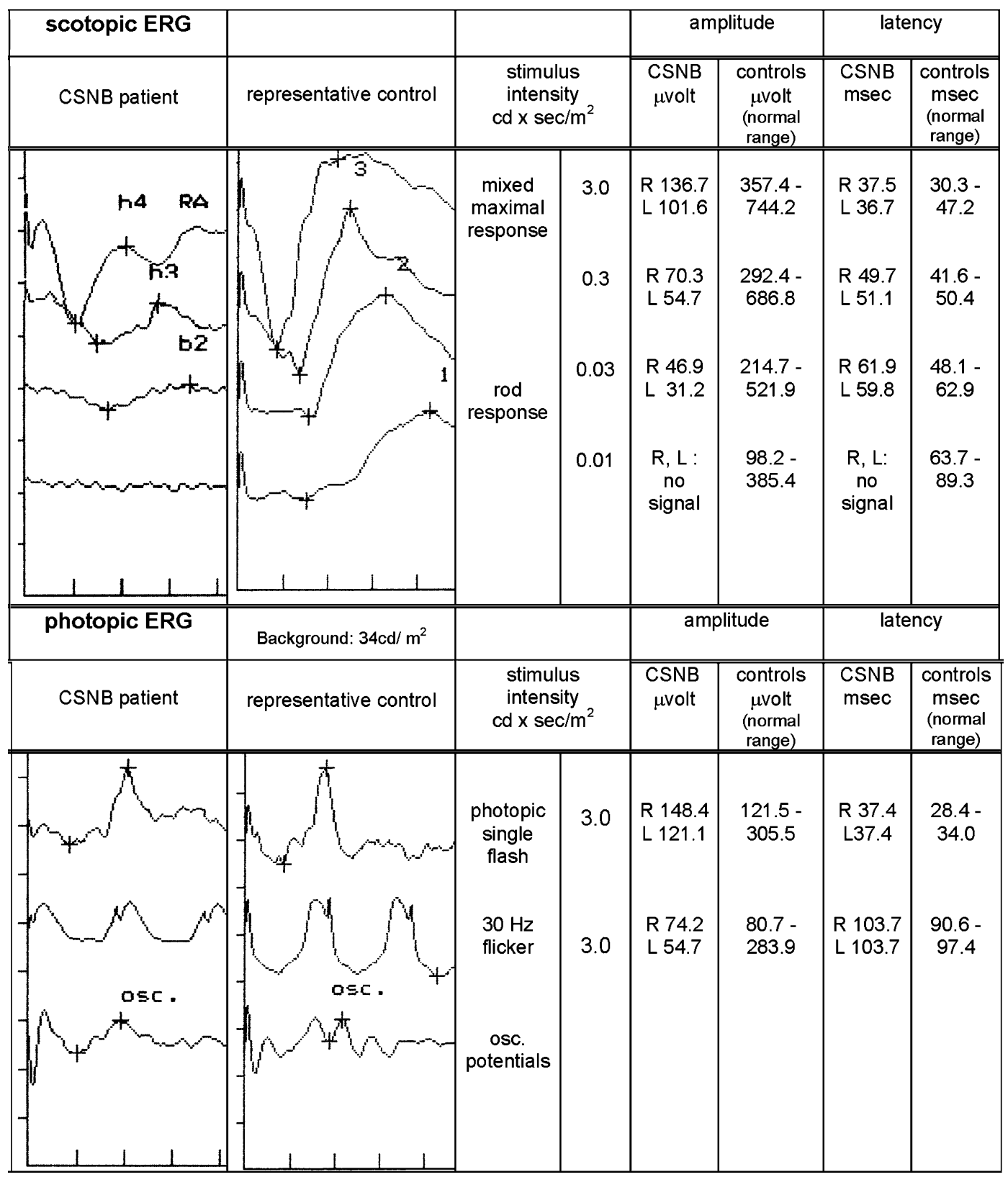

Also, the oscillatory potentials were severely diminished. Because of identical results of both eyes only the results of the right eye are shown. 
shown to result from mutations within the phototransduction cascade (type Nougaret: alpha-subunit of transducin [7], and type Rambusch: beta-subunit of cGMP-phosphodiesterase [8]).

Recently the multifocal ERG has been applied to differentiate on- and off-bipolar-responses through long-duration stimuli generation [9]. In healthy controls an increase of the off-/on-ratio with eccentricity has been documented [9]. Multifocal analysis of patients presenting with cCSNB applying short-duration stimuli revealed changes regarding mainly the P1 implicit times and the N2 wave-forms in the first order kernels, but most of all the overall wave-forms of the second-order kernels [10]. In the Ganzfeld-ERG, stimulation with long-duration stimuli allows an even more pronounced separation between the complete and the incomplete CSNB type when compared to standard ERG recordings [11]. Here we report a multifocal approach in a CSNB patient (complete type) with differentiation of on- and off-responses through long-duration stimuli presentation generated with a LED-screen.

\section{Patient}

A 63-year-old woman presented with night blindness and reduced daytime vision since her early childhood as well as myopic astigmatism ( $\mathrm{R}$ : $\left.-6.0 \mathrm{sph},-3.0 \mathrm{cyl} / 80^{\circ}, \mathrm{L}:-9.0 \mathrm{sph},-2.0 \mathrm{cyl} / 90^{\circ}\right)$. Apart from low degree myopic posterior segment changes and a slight cataract no ocular pathology, especially no nystagm or squinting, was evident. Her best corrected visual acuity was 20/40 (right eye) and 20/50 (left eye). Based on biomicroscopy, electrophysiological and psychophysical examinations she was diagnosed with the complete Schubert-Bornschein type of congenital stationary night blindness: The Ganzfeld-ERG is shown in Table 1 and exhibits a negative-shaped ERG for the mixed maximal rod and cone response, an absent rod response, as well as a slightly reduced amplitude and increased implicit times under photopic conditions (single photopic flash, 30-Hz-flicker). The oscillatory potentials were severely diminished.

The results of the dark adaptometry (Goldmann-Weekers) are seen in Figure 1: A complete absence of the rod response results in a monophasic curve.

\section{Methods}

For multifocal ERG recording both eyes of the patient and one eye of each control $(n=4)$ were examined in maximal mydriasis $(0.5 \%$

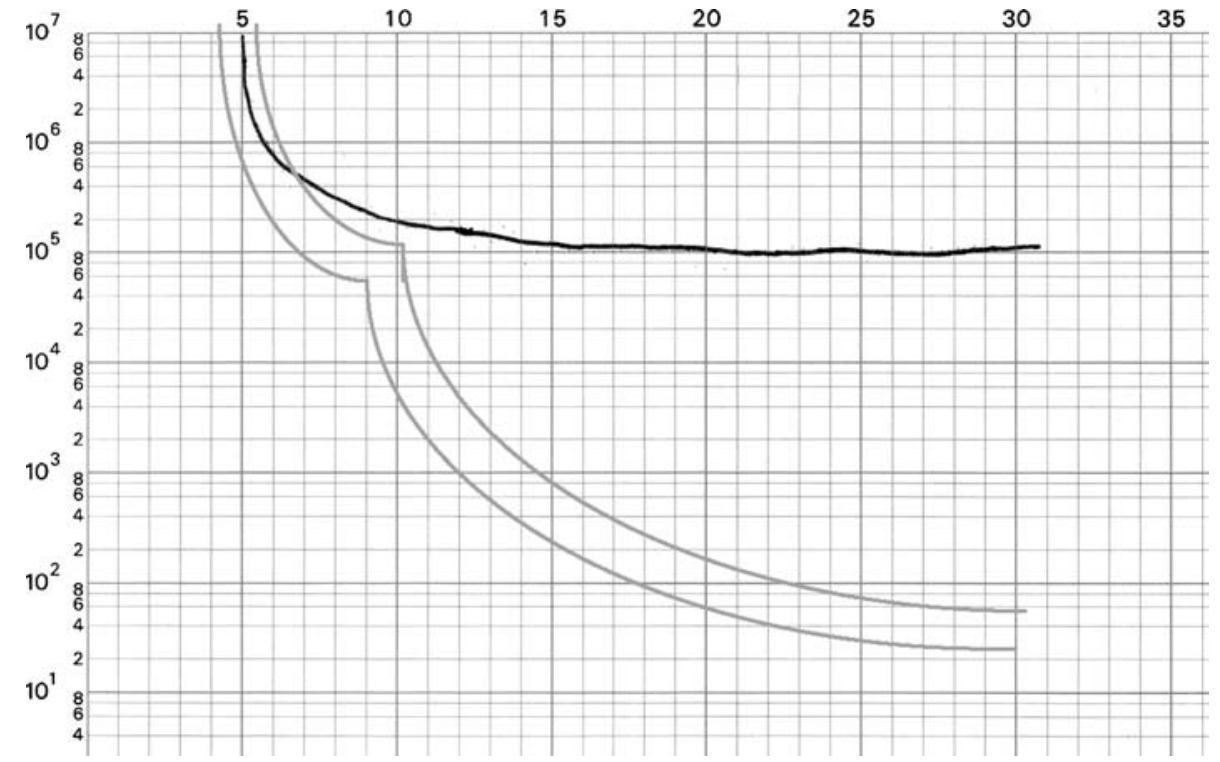

Figure 1. Dark adaption (Goldmann-Weekers) demonstrates complete absence of the rod response resulting in a monophasic curve (black line). Age-matched normal controls would lie within the grey area. 


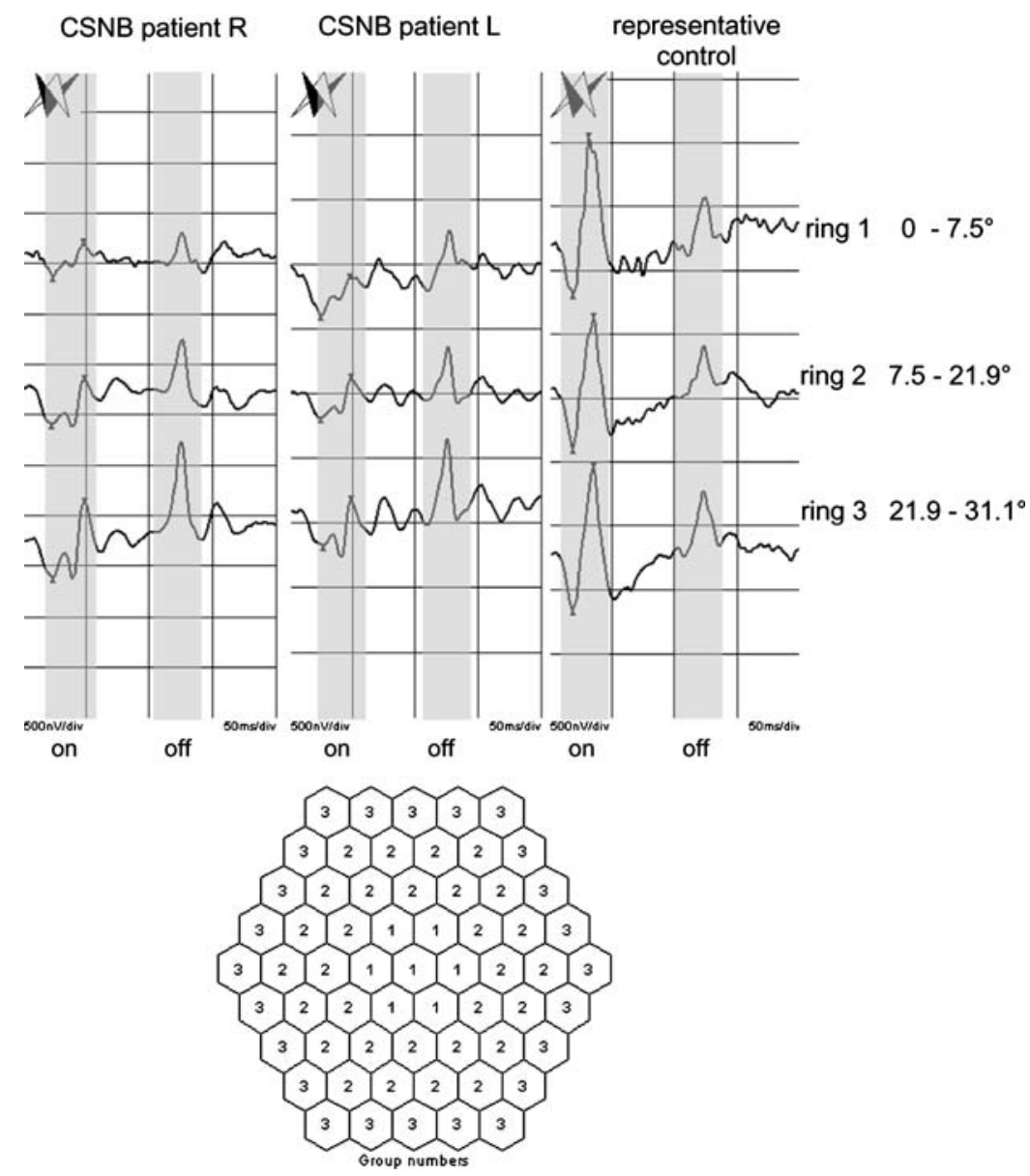

Figure 2. Multifocal ERG on- and off-responses of the CSNB patient (right eye: left column, left eye: middle column) and a representative control (right column) after stimulus presentation with the LED-monitor.

Table 2. Amplitudes of the on- and off-responses and the off/on-ratio of the CSNB patient and controls $(n=4)$

\begin{tabular}{|c|c|c|c|c|c|c|}
\hline \multirow[t]{2}{*}{ Ring area } & \multicolumn{2}{|c|}{$\mathrm{ON}$} & \multicolumn{2}{|c|}{ OFF } & \multicolumn{2}{|c|}{$\mathrm{OFF} / \mathrm{ON}$} \\
\hline & CSNB ( $\mu$ volt) & controls ( $\mu$ volt) & CSNB $(\mu$ volt $)$ & controls ( $\mu$ volt) & ratio $\mathrm{CSNB}$ & ratio controls \\
\hline \multirow[t]{2}{*}{$1\left(0-7.5^{\circ}\right)$} & R 16.6 & $46.2-69$ & R 15.2 & $19.9-28.7$ & R 0.92 & $0.36-0.62$ \\
\hline & L 18.8 & & L 25.0 & & L 1.33 & \\
\hline \multirow[t]{2}{*}{$2\left(7.5-21.9^{\circ}\right)$} & R 12.2 & $24-29$ & R 13.7 & $8.4-14.6$ & R 1.12 & $0.30-0.54$ \\
\hline & L 9.4 & & L 11.3 & & L 1.2 & \\
\hline \multirow[t]{2}{*}{$3\left(21.9-31.1^{\circ}\right)$} & R 13.3 & $20-25$ & R 16.0 & $9.0-12.8$ & $\mathrm{R} 1.2$ & $0.40-0.59$ \\
\hline & L 7.0 & & L 11.2 & & L 1.6 & \\
\hline
\end{tabular}

tropicamide and $0.5 \%$ phenylephrine hydrochloride), while the other eye was occluded. After local anaesthesia $(0.5 \%$ Alcaine eye drops) a bipolar contact lens electrode (GoldLens, Dyagnosys LLC) was inserted. The ground electrode was attached to the forehead. The visual stimulus was generated by a screen of $32 \times 32$ light emitting diodes (luminance: $\left.1(\min )-180(\max ) \mathrm{cd} / \mathrm{m}^{2}\right)$ displayed in a pattern of 61 hexagons. To compensate for different photoreceptor distribution with eccentricity these hexagons were scaled in size. On- and off-phases of $100 \mathrm{~ms}$ each were alternating. MfERGs were recorded with Retiscan ${ }^{\mathrm{TM}}$. The band-pass filter was set at 10-200 Hz. Total 


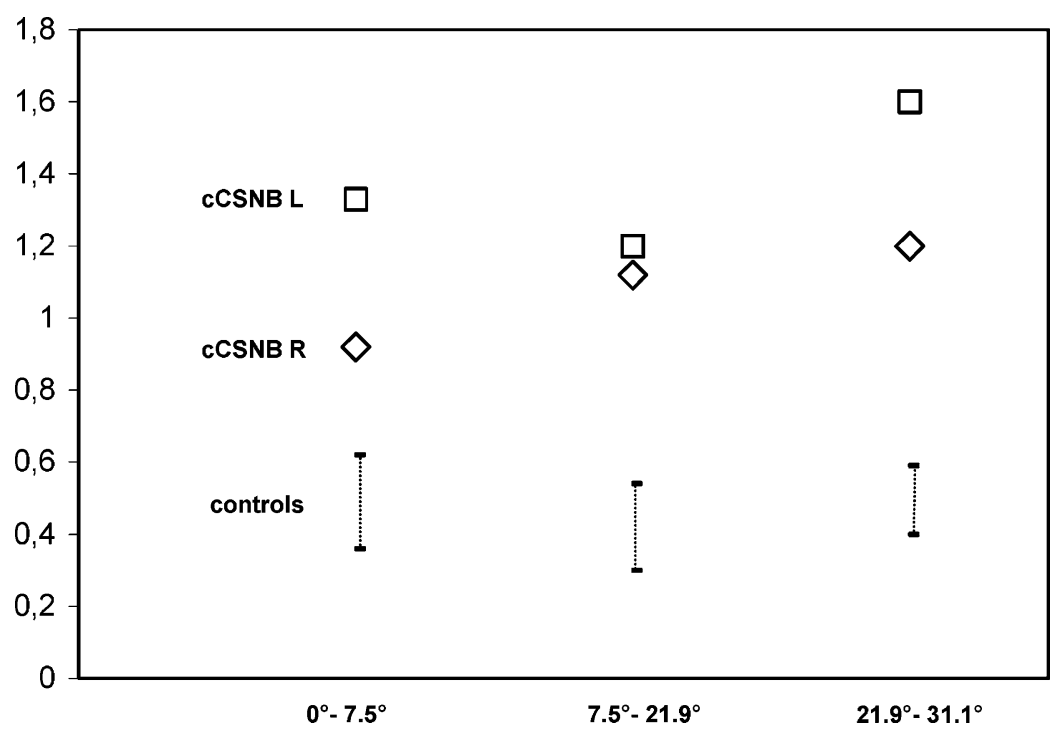

Figure 3. Amplitude off/on-ratio of the CSNB patient and controls $(n=4)$, with the dotted line representing the range of normal. The off/on-ratio of the CSNB patient shows a substantial elevation without any overlap with the controls.

Table 3. off- and on-latencies of the CSNB patient and controls $(n=4)$.

\begin{tabular}{|c|c|c|c|}
\hline \multicolumn{2}{|c|}{$\mathrm{ON}$} & \multicolumn{2}{|c|}{ OFF } \\
\hline $\begin{array}{l}\text { CSNB } \\
\text { ring } 1-3 \mathrm{msec}\end{array}$ & $\begin{array}{l}\text { controls ring } \\
1-3 \mathrm{msec}\end{array}$ & $\begin{array}{l}\text { CSNB } \\
\text { ring } 1-3 \mathrm{msec}\end{array}$ & $\begin{array}{l}\text { controls ring } \\
1-3 \mathrm{msec}\end{array}$ \\
\hline R, L 46.9 & $34.7-38.8$ & $\mathrm{R}, \mathrm{L} 125.5$ & 122.4 \\
\hline
\end{tabular}

recording time was $13.28 \mathrm{~min}$, split up for comfort into 8 periods of $1.46 \mathrm{~min}$ duration. For analysis each data point was averaged with it's two adjacent data points on either side. This smoothing procedure was performed twice.

\section{Results}

Wave forms of the patient's right and left eye and of a representative control are seen in Figure 2. In all eccentricities (ring 1 to 3 ) the amplitudes of the on-responses are severely diminished, while the off-responses are normal (for values see Table 2). To adjust for interindividual amplitude differences the off/on-ratio was calculated. With eccentricity the off/on-ratio tends to show a slight increase in all subjects tested. The off/on-ratio of the CSNB patient shows a substantial elevation without any overlap with the controls (Table 2, Figure 3). The increase with eccentricity is preserved. All latencies are listed in Table 3: The implicit time of the
P1-off-response was normal in the CSNB patient when compared to controls, whereas the P1 of the on-response showed a delay in implicit time (46.9 ms, normal range: $34.7-38.8 \mathrm{~ms}$ ). Again this was found for all eccentricities.

\section{Discussion}

Applying a multifocal ERG approach with longduration stimuli facilitated the classification of our patient to one of the CSNB subtypes and supported the original diagnose that was based on standard ERG results. The homogenous pattern of amplitude reduction in all eccentricities seems to correlate to the congenital type of this disorder that involves on-bipolar cells evenly across the retina. In contrast, one of our patients with an acquired retinal damage (MAR) failed to show such a symmetrical damage pattern (data presented at the $102^{\text {nd }}$ meeting of the German Academy of Ophthalmology, Berlin). As in standard ERG-testing, implicit times of the multifocal ERG responses generated with long-duration stimuli were affected as well. Separating the onund off-responses suggests that the increase in implicit time seen in standard ERG recordings of CSNB patients is a characteristic of the on-response, since all off-response implicit times were normal (in all eccentricities). In context of the fast-stimulation mfERG findings in CSNB (see 
introduction), the responses of the slow-stimulation mfERG in CSNB reported here are in support of the findings by Hood et al. [12]. They postulated that the P1 peak in fast-stimulation mfERG testing is composed of a positive on-peak, representing the on-bipolar response, partially superimposed by a shortly delayed positive offpeak, representing the off-bipolar response. With increasing stimuli length these two positive peaks separate and can be examined independently. The use of a LED screen seems to be a suitable approach, because it provides the advantage of stable luminance during the long-duration stepshaped on-stimulus.

\section{Acknowledgement}

Supported by a grand from Pfizer (MT, AP)

\section{References}

1. Miyake Y, Yagasaki K, Horiguchi M, Kawase Y, Kanda T. Congenital stationary night blindness with negative electroretinogram. A new classification. Arch Ophthalmol, 1986; 104(7): 1013-20.

2. Sandberg MA, Pawlyk BS, Dan J, Arnaud B, Dryja TP, Berson EL. Rod and cone function in the Nougaret form of stationary night blindness. Arch Ophthalmol, 1998; 116(7): 867-72.

3. Rosenberg T, Haim M, Piczenik Y, Simonsen SE. Autosomal dominant stationary night-blindness. A large family rediscovered. Acta Ophthalmol (Copenhagen), 1991; 69(6): 694-702.

4. Pusch CM, Zeitz C, Brandau O, Pesch K, Achatz H, Feil S, Scharfe C, Maurer J, Jacobi FK, Pinckers A, Andreasson S, Hardcastle A, Wissinger B, Berger W, Meindl A. The complete form of $\mathrm{X}$-linked congenital stationary night blindness is caused by mutations in a gene encoding a leucine-rich repeat protein. Nat Genet, 2000; 26(3): 324-7.

5. Bech-Hansen NT, Naylor MJ, Maybaum TA, Sparkes RL, Koop B, Birch DG, Bergen AA, Prinsen CF, Polomeno RC, Gal A, Drack AV, Musarella MA, Jacobson SG, Young RS, Weleber RG. Mutations in NYX, encoding the leucine-rich proteoglycan nyctalopin, cause X-linked complete congenital stationary night blindness. Nat Genet, 2000; 26(3): 319-23.

6. Jacobi FK, Hamel CP, Arnaud B, Blin N, Broghammer M, Jacobi PC, Apfelstedt-Sylla E, Pusch CM. A novel CACNA1F mutation in a french family with the incomplete type of X-linked congenital stationary night blindness. Am J Ophthalmol, 2003; 135(5): 733-6.

7. Muradov KG, Artemyev NO. Loss of the effector function in a transducin-alpha mutant associated with Nougaret night blindness. J Biol Chem, 2000; 275(10): 6969-74.

8. Muradov KG, Granovsky AE, Artemyev NO. Mutation in rod PDE6 linked to congenital stationary night blindness impairs the enzyme inhibition by its gamma-subunit. Biochemistry, 2003; 42(11): 3305-10.

9. Kondo M, Miyake Y, Horiguchi M, Suzuki S, Tanikawa A. Recording multifocal electroretinogram on and off responses in humans. Invest Ophthalmol Vis Sci, 2003 Mar; 39(3): 574-80.

10. Kondo M, Miyake Y, Kondo N, Tanikawa A, Suzuki S, Horiguchi M, Terasaki H. Multifocal ERG findings in complete type congenital stationary night blindness. Invest Ophthalmol Vis Sci, 2001; 42(6): 1342-8.

11. Langrova H, Gamer D, Friedburg C, Besch D, Zrenner E, Apfelstedt-Sylla E. Abnormalities of the long flash ERG in congenital stationary night blindness of the Schubert-Bornschein type. Vision Res, 2002; 42(11): 1475-83.

12. Hood DC, Frishman LJ, Saszik S, Viswanathan S. Retinal origins of the primate multifocal ERG: implications for the human response. Invest Ophthalmol Vis Sci, 2002; 43(5): 1673-85.

Address for correspondence: Dorothee Leifert, Department of Ophthamlology, University Hospital Basel, Mittlere Strasse 91, CH-4056, Basel, Switzerland

Phone: +41-61-265-8787; Fax: +41-61-265-8740; E-mail: leifertd@uhbs.ch) 